\title{
Remarks on $r$-planes in Complete Intersections
}

\author{
Chikashi MIYAZAKI \\ Kumamoto University \\ (Communicated by H. Kaji)
}

\begin{abstract}
This paper investigates the families of smooth complete intersections containing $r$-planes in projective spaces. We are going in a primitive way to shed some light on a point and an $r$-plane containing the point in a complete intersection from the viewpoint of projective geometry.
\end{abstract}

\section{Introduction}

This paper investigates the families of smooth complete intersections containing $r$-planes in the projective space. We will study a classical Fano scheme, for example, the one which parametrizes pairs of a point $z$, an $r$-plane $L$ and a complete intersection $V$ satisfying that $z$ is a point of $L$ and $L$ is contained in $V$. On one hand, there has been some references, e.g., $[3,4,9]$, on this topic arising from the unirationality problem for specific projective varieties. On the other hand, we find a classical way to study an expected dimension of Fano schemes as in $[2,5,10,11]$.

The purpose of this paper, mostly inspired by [2], is to give a primitive way to grasp the relations between a point, an $r$-plane and a complete intersection in terms of a generalized normal sheaf coming from Hilbert scheme.

In Section 2, we introduce a generalized normal sheaf whose section gives a tangent space of an incidence correspondence. The infinitesimal theory presented here is given rise from a detailed proof of [2] and will be used to prove the main results of this paper in Section 3.

Let $\mathbf{P}_{k}^{N}$ be the projective space over an algebraically closed field $k$. Let $n, r$ and $d_{1}, \ldots, d_{N-n}$ be integers with $2 \leq n \leq N-1$, and $1 \leq r \leq n / 2$ and $d_{j} \geq 2$ for $j=1, \ldots, N-n$. Now we pose the following questions: (1) If $V$ is a complete intersection of $\left(d_{1}, \ldots, d_{N-n}\right)$ of $\mathbf{P}_{k}^{N}$, is there an $r$-plane contained in $V$ ? (2) Further, for any point $z$ of $V$, is there an $r$-plane contained in $V$ through the point $z$ ? (3) Let $H$ be the scheme parametrizing the complete intersections of $\left(d_{1}, \ldots, d_{N-n}\right)$ of $\mathbf{P}_{k}^{N}$. Let $H_{r}$ be the subscheme of $H$ which parametrizes the complete intersections containing an $r$-plane of $\mathbf{P}_{k}^{N}$. Then $H_{0}$

Received May 13, 2015; revised August 21, 2015

2000 Mathematics Subject Classification: 14C05, 14M10

Partially supported by Grant-in-Aid for Scientific Research (C) (26400048) Japan Society for the Promotion of Science 
is an open set of $H$, and $H_{r}$ is a closed subscheme of $H_{0}$. When is $H_{r}$ nonempty? What is $\operatorname{dim} H_{r}$ ?

As references mentioned before, several results has been somewhat investigated since the time of [10], which gives, in fact, an answer for (1). As far as I know, however, there are no written statements concerning (2) and (3). We will give answers concerning (2) and (3) in Section 3, see below, with presenting transparent view in the process of Theorem 3.1 and Theorem 3.3.

COROllary 1.1. Let $V$ be a complete intersection of type $\left(d_{1}, \ldots, d_{N-n}\right)$ in $\mathbf{P}_{k}^{N}$. If $(r+1)(N-r) \geq \sum_{j=1}^{N-n}\left(\begin{array}{c}r+d_{j} \\ r\end{array}\right)+n-r$ and $n \geq 2 r$, then for any point $z$ of $V$ there is an $r$-plane through the point $z$ and lying on $V$.

COROLLARY 1.2. Let $H_{r}$ be a scheme which parametrizes the smooth complete intersection of type $\left(d_{1}, \ldots, d_{N-n}\right)$ containing an $r$-plane of $\mathbf{P}_{k}^{N}$. Then $H_{r}$ is nonempty if and only if $n \geq 2 r$. Further, $\operatorname{dim} H_{0}=\sum_{j=1}^{N-n}\left(\begin{array}{c}n+d_{j} \\ d_{j}\end{array}\right)$, and $H_{r}$ is a unirational subvariety of $H_{0}$ with $\operatorname{codim}\left(H_{r}, H_{0}\right)=\max \left\{\sum_{j=1}^{N-n}\left(\begin{array}{c}r+d_{j} \\ r\end{array}\right)-(r+1)(N-r), 0\right\}$.

\section{Infinitesimal Theory}

In this section, we will develop infinitesimal theory for a ladder of subschemes in an ambient space.

Definition 2.1. Let $Y_{1}, \ldots, Y_{n}$ be local complete intersections in a scheme $P$ with $Y_{1} \subset \cdots Y_{n} \subset P$. For a normal sheaf $\mathcal{N}_{Y_{i} / P}=\left(\mathcal{I}_{Y_{i} / P} / \mathcal{I}_{Y_{i} / P}^{2}\right)^{\vee}$ we consider natural morphisms $\alpha_{i}:\left.\mathcal{N}_{Y_{i} / P} \rightarrow \mathcal{N}_{Y_{i+1} / P}\right|_{Y_{i}}$ for $i=1, \ldots, n-1$ and $\beta_{i}:\left.\mathcal{N}_{Y_{i} / P} \rightarrow \mathcal{N}_{Y_{i} / P}\right|_{Y_{i-1}}$ for $i=2, \ldots, n$. Then we define a generalized normal sheaf $\mathcal{N}_{Y_{1} / \cdots / Y_{n} / P}$ of $Y_{1} \subset \cdots \subset Y_{n}$ in $P$ to be the sheaf $\operatorname{Ker} \gamma$, where

$$
\gamma=\left(\begin{array}{ccccccc}
\alpha_{1} & -\beta_{2} & 0 & 0 & \ldots & \ldots & 0 \\
0 & \alpha_{2} & -\beta_{3} & 0 & \ldots & \ldots & 0 \\
\ldots & \cdots & \ldots & \ldots & \ldots & \ldots & \ldots \\
0 & \ldots & \ldots & \ldots & 0 & \alpha_{n-1} & -\beta_{n}
\end{array}\right):\left.\oplus_{i=1}^{n} \mathcal{N}_{Y_{i} / P} \rightarrow \oplus_{j=2}^{n} \mathcal{N}_{Y_{j} / P}\right|_{Y_{j-1}} .
$$

REMARK 2.2. For $n=2$ we take $L=Y_{1}$ and $V=Y_{2}$. Then a generalized normal sheaf $\mathcal{N}_{L / V / P}$ on $P$ can be regarded as the fibre product:

$$
\begin{array}{ccc}
\mathcal{N}_{L / V / P} & \rightarrow & \mathcal{N}_{V / P} \\
\downarrow & \square & \downarrow \\
\mathcal{N}_{L / P} & \rightarrow & \left.\mathcal{N}_{V / P}\right|_{L} .
\end{array}
$$

LEMMA 2.3. Let $R$ be a Noetherian ring and $\mathfrak{a}$ be an ideal of $R$ with $\mathfrak{a}^{2}=0$. Let $\bar{R}=$ $R / \mathfrak{a}$. Let $P$ be a scheme flat over $R$ and $\bar{P}=P \times_{R} \bar{R}$. Let $\bar{Y}_{1} \subset \cdots \subset \bar{Y}_{n}$ be local complete intersections in $\bar{P}$, which are flat over $\bar{R}$. Let us take $\varphi: \bar{Y}_{1} \subset \cdots \subset \bar{Y}_{n} \subset \bar{P} \rightarrow \operatorname{Spec} \bar{R}$. 
(1) Assume that $\varphi$ is locally liftable over $R$. Then there is a canonical class of obstruction in $\mathrm{H}^{1}\left(\mathcal{N}_{\bar{Y}_{1} / \cdots / \bar{Y}_{n} / \bar{P}} \otimes_{\bar{R}} \mathfrak{a}\right)$, which is zero if and only if $\varphi$ is globally liftable over $R$.

(2) If $\varphi$ is liftable, then the set of liftings over $R$ is a principal homogeneous set under $\mathrm{H}^{0}\left(\mathcal{N}_{\bar{Y}_{1} / \cdots / \bar{Y}_{n} / \bar{P}} \otimes_{\bar{R}} \mathfrak{a}\right)$.

In case $n=1$, Lemma 2.3 is a consequence of [6, Exposé 221].

Proof. Let us prove for $n=2$. It is similarly proved for $n \geq 3$. Let $\bar{L}=\bar{Y}_{1}$ and $\bar{V}=$ $\bar{Y}_{2}$. First we will prove (2). Since $\varphi$ is liftable from the assumption, there is a lifting $L_{1} \subset V_{1}$ in $P$. We show that there is one-to-one correspondence between the set of liftings in $P$ and the elements of $\mathrm{H}^{0}\left(\mathcal{N}_{\bar{L} / \bar{V} / \bar{P}} \otimes_{\bar{R}} \mathfrak{a}\right)$ such that the fixed lifting $L_{1} \subset V_{1}$ corresponds to 0 as an element of $\mathrm{H}^{0}\left(\mathcal{N}_{\bar{L} / \bar{V} / \bar{P}} \otimes_{\bar{R}} \mathfrak{a}\right)$. By [1,7] a pair of lifting $L$ and $V$ in $P$ without the condition $L \subset V$ corresponds to an element $(\psi, \phi)$ of $\mathrm{H}^{0}\left(\mathcal{N}_{\bar{L} / \bar{P}} \otimes_{\bar{R}} \mathfrak{a}\right) \oplus \mathrm{H}^{0}\left(\mathcal{N}_{\bar{V} / \bar{P}} \otimes_{\bar{R}} \mathfrak{a}\right)$. So we have only to show that $L$ is contained in $V$ if and only of $(\psi, \phi)$ belongs to $\mathrm{H}^{0}\left(\mathcal{N}_{\bar{L} / \bar{V} / \bar{P}} \otimes_{\bar{R}} \mathfrak{a}\right)$, where $\mathrm{H}^{0}\left(\mathcal{N}_{\bar{L} / \bar{V} / \bar{P}} \otimes_{\bar{R}} \mathfrak{a}\right)$ is considered as a fibre product of $\mathrm{H}^{0}\left(\mathcal{N}_{\bar{L} / \bar{P}} \otimes_{\bar{R}} \mathfrak{a}\right)$ and $\mathrm{H}^{0}\left(\mathcal{N}_{\bar{V} / \bar{P}} \otimes_{\bar{R}} \mathfrak{a}\right)$ over $\mathrm{H}^{0}\left(\left.\mathcal{N}_{\bar{V} / \bar{P}}\right|_{L} \otimes_{\bar{R}} \mathfrak{a}\right)$, see Remark 2.2.

The question is local. Let $x$ be a point of $P$. We set $\bar{A}=\mathcal{O}_{\bar{P}, x}, \bar{I}=\mathcal{I}_{\bar{V} / \bar{P}, x}, \bar{J}=$ $\mathcal{J}_{L / P, x}, A=\mathcal{O}_{P, x}, I_{1}=\mathcal{I}_{V_{1} / P, x}, J_{1}=\mathcal{J}_{L_{1} / P, x}, I=\mathcal{I}_{V / P, x}, J=\mathcal{J}_{L / P, x}, B_{1}=A / I_{1}$, $C_{1}=A / J_{1}, \bar{B}=\bar{A} / \bar{I}$ and $\bar{C}=\bar{A} / \bar{J}$. Keeping in mind that $L$ and $V$ are local complete intersections, we put that $I_{1}=\left(f_{1}, \ldots, f_{r}\right), I=\left(f_{1}+g_{1}, \ldots, f_{r}+g_{r}\right), J_{1}=\left(p_{1}, \ldots, p_{s}\right)$ and $J=\left(p_{1}+q_{1}, \ldots, p_{s}+q_{s}\right)$ by minimal generators in the ring $A$. Then $\phi$ can be locally written as an $\bar{A}$-homomorphism from $\bar{I}$ to $\bar{B} \otimes_{\bar{R}} \mathfrak{a}$ by $\phi\left(f_{i}\right)=g_{i}$ for $i=1, \ldots, r$, where $f_{i}$ and $g_{i}$ are images in $\bar{I}$ and $\bar{B} \otimes_{\bar{R}} \mathfrak{a}$ respectively by abuse of notation. Similarly, $\psi$ can be regarded as an $\bar{A}$-homomorphism from $\bar{J}$ to $\bar{C} \otimes_{\bar{R}} \mathfrak{a}$ by $\phi\left(p_{i}\right)=q_{i}$ for $i=1, \ldots, r$.

Comparing with the diagram in Remark 2.2 , we locally consider the fibre product of $\alpha: \operatorname{Hom}_{\bar{C}}\left(\bar{J} / \bar{J}^{2}, \bar{C} \otimes_{\bar{R}} \mathfrak{a}\right) \rightarrow \operatorname{Hom}_{\bar{C}}\left(\bar{J} / \bar{I} \bar{J}, \bar{C} \otimes_{\bar{R}} \mathfrak{a}\right)$ and $\beta: \operatorname{Hom}_{\bar{B}}\left(\bar{I} / \bar{I}^{2}, \bar{B} \otimes_{\bar{R}} \mathfrak{a}\right) \rightarrow$ $\operatorname{Hom}_{\bar{C}}\left(\bar{I} / \bar{I} \bar{J}, \bar{C} \otimes_{\bar{R}} \mathfrak{a}\right)$. Thus we have only to show that $I \subset J$ if and only if $\alpha(\psi)=\beta(\phi)$. Note that $I \subset J$ means that $f_{i}+g_{i}=\sum_{j} a_{i j}\left(p_{j}+q_{j}\right)$ for some $a_{i j} \in A$. Since $I_{1} \subset J_{1}$, we can take $f_{i}=\sum_{j} a_{i j} p_{j}$ for some $a_{i j} \in A$. Now we have $\alpha(\psi)\left(f_{i}\right)=\alpha(\psi)\left(\sum_{j} a_{i j} p_{j}\right)=$ $\sum_{j} a_{i j} \overline{\psi\left(p_{j}\right)}=\sum_{j} a_{i j} \bar{q}_{j}$ and $\beta(\phi)\left(f_{i}\right)=\overline{\phi\left(f_{i}\right)}=\bar{g}_{i}$ in $\bar{C} \otimes_{\bar{R}} \mathfrak{a}$, where $\overline{\psi\left(p_{j}\right)}, q_{j}, \overline{\phi\left(f_{i}\right)}$ and $\bar{g}_{i}$ are the images in $\bar{C} \otimes_{\bar{R}}$ a. Hence the assertion is proved. Moreover, (1) immediately follows from the proof of (2).

Let $P=\mathbf{P}_{k}^{N}$ be the projective space over an algebraically closed field $k$. Let $G$ and $H$ be Hilbert schemes parametrizing closed subschemes of $\mathbf{P}_{k}^{N}$. Then we construct a scheme $X$ which parametrizes pairs $(L, V)$ of closed subschemes of $\mathbf{P}_{k}^{N}$ such that $L \subset V$. It is a closed subscheme of $G \times H$, called an incidence correspondence. 
PROPOSITION 2.4. Under the above conditions, let $x$ be a point of $X$ corresponding to $(L, V)$. Then

(1) $T_{x, X}=\Gamma\left(\mathcal{N}_{L / V / P}\right)$.

(2) If $L$ and $V$ are local complete intersections of $V$ and $P$ respectively and $\mathrm{H}^{1}\left(\mathcal{N}_{L / V / P}\right)=0$, then $X$ is smooth at $x$.

PROOF. It immediately follows from Lemma 2.3.

COROllary 2.5. Under the same conditions as in Proposition 2.4, let $f: X \subset G \times$ $H \rightarrow H$. Assume that $\Gamma\left(\mathcal{N}_{V / P}\right) \rightarrow \Gamma\left(\left.\mathcal{N}_{V / P}\right|_{L}\right)$ is surjective. Then $T_{f, x}: T_{x, X} \rightarrow T_{f(x), H}$ is surjective if and only if $\Gamma\left(\mathcal{N}_{L / P}\right) \rightarrow \Gamma\left(\left.\mathcal{N}_{V / P}\right|_{L}\right)$ is surjective. In this case we have $\operatorname{dim} T_{x, X}=\mathrm{h}^{0}\left(\mathcal{N}_{V / P}\right)+\mathrm{h}^{0}\left(\mathcal{N}_{L / P}\right)-\mathrm{h}^{0}\left(\left.\mathcal{N}_{V / P}\right|_{L}\right)$.

In case $L$ is a line and $V$ is a hypersurface of $P=\mathbf{P}_{\mathbf{C}}^{N}$, smooth along $L$, this is Corollary 4 in [2].

PROOF. The corollary follows from Proposition 2.4 and the fibre product diagram:

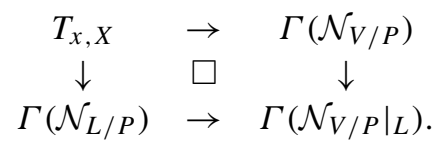

\section{3. $r$-planes in complete intersections}

In this section, we will investigate a complete intersection which contains $r$-planes (through any point of the complete intersection). Let $k$ be an algebraically closed field. Let $r, n$ and $N$ be integers with $0 \leq r<n<N$. Let $H$ be a scheme which parametrizes the complete intersections of type $\left(d_{1}, \ldots, d_{N-n}\right)$ in the projective space $\mathbf{P}_{k}^{N}$ over $k$, where $2 \leq d_{1} \leq \cdots \leq d_{N-n}$. Let us put $\left(d_{1}, \ldots, d_{N-n}\right)=\left(e_{1}, \ldots, e_{p}\right)$, where $e_{1}<\cdots<e_{p}$, $\ell_{0}=0$ and $e_{i}=d_{\ell_{i-1}+1}=\cdots=d_{\ell_{i}}$ for $i=1, \ldots, p$, that is, $\ell_{i}$ is the number of times in which $e_{i}$ appears in the sequence $\left(d_{1}, \ldots, d_{N-n}\right)$. Let $G_{e_{1} \cdots e_{j}}$ be the scheme of the complete intersections of type $\left(d_{1}, \ldots, d_{\ell_{1}+\cdots+\ell_{j}}\right)$ from the construction of Hilbert scheme, see, e.g., [8]. Then we easily see that $G_{e_{1}}$ is isomorphic to the Grassmann scheme $G\left(\ell_{1}, m_{1}\right)$, where $m_{1}=\operatorname{dim} \Gamma\left(\mathcal{O}_{\mathbf{P}_{k}^{N}}\left(e_{1}\right)\right)$. As a Hilbert scheme we inductively have a natural map $\varphi_{j}: G_{e_{1} \cdots e_{j}} \rightarrow G_{e_{1} \cdots e_{j-1}}$, which is a Grassmann bundle. In particular, a fibre of $x \in G_{e_{1} \cdots e_{j-1}}$ corresponding to a complete intersection $V$ of type $\left(d_{1}, \ldots, d_{\ell_{1}+\cdots+\ell_{i-1}}\right)$ is isomorphic to $G\left(\ell_{i}, m_{i}\right)$, where $m_{i}=\operatorname{dim} \Gamma\left(\mathcal{O}_{V}\left(e_{i}\right)\right)$. In this way we have a sequence of Grassmann bundles $H=G_{e_{1} \cdots e_{p}} \rightarrow G_{e_{1} \cdots e_{p-1}} \rightarrow \cdots \rightarrow G_{e_{1}} \rightarrow$ Spec $k$, which especially gives that $H$ is rational. Let $G=G(r+1, N+1)$ be the Grassmann scheme of $r$-planes in $\mathbf{P}_{k}^{N}$. Then we will consider a family of the complete intersections containing $r$-planes. Let us put an incidence correspondence $X=\{(L, V) \in G \times H \mid L \subset V\}$, and write $f: X \subset G \times H \rightarrow H$ and 
$g: X \subset G \times H \rightarrow G$. For $X_{e_{1} \cdots e_{j}}=X \cap\left(G \times G_{e_{1} \cdots e_{j}}\right)$ in $G \times H$, we have a sequence of Grassmann bundles $X=X_{e_{1} \cdots e_{p}} \rightarrow X_{e_{1} \cdots e_{p-1}} \rightarrow \cdots \rightarrow X_{e_{1}} \rightarrow G$, which especially gives that $X$ is rational. In this case we have

$$
\operatorname{dim} X-\operatorname{dim} H=(r+1)(N-r)-\sum_{j=1}^{N-n}\left(\begin{array}{c}
r+d_{j} \\
r
\end{array}\right) .
$$

From now on we always assume that $n \geq 2 r$ from the viewpoint of Remark 3.4.

THEOREM 3.1. Under the above conditions, we have

(1) If $\operatorname{dim} X \geq \operatorname{dim} H$, then $f$ is surjective.

(2) If $\operatorname{dim} X \leq \operatorname{dim} H$, then $\operatorname{dim} X=\operatorname{dim} f(X)$.

Proof. The assertion (1) has been already given by Predonzan [10], see also Borcea [4]. The proof is similarly done as in Theorem 3.3 except for the hyperquadrics. For $n=N-1$ and $d_{1}=2$ we will prove that $f$ is surjective even for char $k=2$. It suffices to find a point $x \in X$ where $T_{f, x}: T_{x, X} \rightarrow T_{f(x), H}$ is surjective. Let $\mathbf{P}_{k}^{N}=$ Proj $k\left[X_{0}, \ldots, X_{N}\right]$. By taking $L: X_{r+1}=\cdots=X_{N}=0$ and $V: F\left(X_{0}, \ldots, X_{N}\right)=0$, where $F\left(X_{0}, \ldots, X_{N}\right)=X_{0} X_{N}+\cdots+X_{(N-1) / 2} X_{(N+1) / 2}$ if $N$ is odd, $F\left(X_{0}, \ldots, X_{N}\right)=$ $X_{0} X_{N-1}+\cdots+X_{(N-2) / 2} X_{N / 2}+X_{N}^{2}$ if $N$ is even, we want to show that $T_{f, x}$ is surjective at the point $x \in X$ corresponding to $(L, V)$. In fact, it is equivalent to saying that $\varphi: \Gamma\left(\mathcal{N}_{L / P}\right) \rightarrow \Gamma\left(\left.\mathcal{N}_{V / P}\right|_{L}\right)$ is surjective by Corollary 2.5 , which is explicitly written as a map $\left[\partial /\left.\partial X_{r+1}\right|_{L}, \ldots, \partial /\left.\partial X_{N}\right|_{L}\right]: \oplus^{N-r} \Gamma\left(\mathcal{O}_{L}(1)\right) \rightarrow \Gamma\left(\mathcal{O}_{L}(2)\right)$ by [2], that is, $\varphi=\left[0, \ldots, 0, X_{r}, \ldots, X_{0}\right]$ if $N$ is odd, $\varphi=\left[0, \ldots, 0, X_{r}, \ldots, X_{0}, 0\right]$ if $N$ is even. Hence we have done.

Now we will prove (2). Set $\varepsilon=\operatorname{dim} X-\operatorname{dim} f(X)$. Let us take an $r$-plane $L \in G$. Then $H^{\prime}=f\left(g^{-1}(L)\right)$ is a subvariety of $H$ with $\operatorname{dim} H^{\prime}=\operatorname{dim} X-\operatorname{dim} G$. There is an irreducible component $Y$ of $f^{-1}\left(H^{\prime}\right)$ such that $f(Y)=H^{\prime}$ and $\operatorname{dim} Y=\operatorname{dim} f^{-1}\left(H^{\prime}\right)$. So we have $\operatorname{dim} Y \geq \operatorname{dim} X-\operatorname{dim} G+\varepsilon$. Let $g^{\prime}: Y \rightarrow G^{\prime}=g(Y)(\subseteq G)$ be a restriction of $g$. Let $G_{s}$ be a closed subscheme of $G$ which parametrizes $r$-planes $L^{\prime}$ such that $\operatorname{dim} L \cap L^{\prime} \geq s$ for an integer $s \geq-1$, where $\operatorname{dim} \phi=-1$. Let $s$ be the maximal integer such that $G^{\prime}$ is contained in $G_{s}^{\prime}$. Fix $L^{\prime} \in G^{\prime}$ such that $\operatorname{dim} L \cap L^{\prime}=s$. Let $S$ be a closed subscheme of $H$ which parametrizes complete intersections containing $L$ and $L^{\prime}$. In case $s=-1$ we have

$$
\begin{aligned}
\operatorname{dim} S & =\operatorname{dim} \operatorname{Ker}\left(\Gamma\left(V, \mathcal{N}_{V / P}\right) \rightarrow \Gamma\left(L,\left.\mathcal{N}_{V / P}\right|_{L}\right) \oplus \Gamma\left(L^{\prime},\left.\mathcal{N}_{V / P}\right|_{L^{\prime}}\right)\right) \\
& =\operatorname{dim} H-2 \mathrm{~h}^{0}\left(V,\left.\mathcal{N}_{V / P}\right|_{L}\right)=2 \operatorname{dim} X-2 \operatorname{dim} G-\operatorname{dim} H
\end{aligned}
$$

by Proposition 2.4 and Corollary 2.5. Thus we see $\operatorname{dim} Y \leq \operatorname{dim} g^{\prime-1}\left(L^{\prime}\right)+\operatorname{dim} G \leq \operatorname{dim} S+$ $\operatorname{dim} G=2 \operatorname{dim} X-\operatorname{dim} G-\operatorname{dim} H$, conclusively $\operatorname{dim} X-\operatorname{dim} G+\varepsilon \leq 2 \operatorname{dim} X-\operatorname{dim} G-$ $\operatorname{dim} H$. So we have $\varepsilon \leq \operatorname{dim} X-\operatorname{dim} H$. Hence we obtain $\varepsilon=0$. For $s \geq 0$ we have

$$
\operatorname{dim} S=\operatorname{dim} \operatorname{Ker}\left(\Gamma\left(V, \mathcal{N}_{V / P}\right) \rightarrow \Gamma\left(L \cup L^{\prime},\left.\mathcal{N}_{V / P}\right|_{L \cup L^{\prime}}\right)\right)
$$




$$
=2 \operatorname{dim} X-2 \operatorname{dim} G-\operatorname{dim} H+\sum_{j=1}^{N-n}\left(\begin{array}{c}
s+d_{j} \\
s
\end{array}\right) .
$$

Also, we see $\operatorname{dim} G_{s}=\operatorname{dim} G-(s+1)(N-2 r+s)$. Hence $\operatorname{dim} Y \leq 2 \operatorname{dim} X-\operatorname{dim} G-$ $\operatorname{dim} H-P(s)$, where $P(s)=(s+1)(N-2 r+s)-\sum_{j=1}^{N-n}\left(\begin{array}{c}s+d_{j} \\ s\end{array}\right)$. Comparing with the proof for $s=-1$, in order to show $\varepsilon=0$ we have only to prove that $P(s) \geq$ $\operatorname{dim} X-\operatorname{dim} H$ for $s=0, \ldots, r$. We have clearly $P(0)=n-2 r \geq 0$ and $P(r)=(r+1)(N-r)-\sum_{j=1}^{N-n}\left(\begin{array}{c}r+d_{j} \\ r\end{array}\right)=\operatorname{dim} X-\operatorname{dim} H$. Also, we see $\Delta P(s)=$ $P(s+1)-P(s)=2 s+N-2 r+2-\sum_{j=1}^{N-n}\left(\begin{array}{c}s+d_{j} \\ s+1\end{array}\right)$, so $\Delta^{2} P(s)=2-$ $\sum_{j=1}^{N-n}\left(\begin{array}{c}s+d_{j} \\ s+2\end{array}\right) \leq 2-\sum_{j=1}^{N-n}\left(\begin{array}{c}d_{j} \\ 2\end{array}\right) \leq 0$. Thus we obtain $P(s) \geq \operatorname{dim} X-\operatorname{dim} H$ for $s=0, \ldots, r$. Hence the assertion is proved.

In the case of (2) in Theorem 3.1, I wonder if $f$ is birational for $d_{i} \gg 0$. Anyway we move on showing a more or less known result in Proposition 3.2. Our proof is effective even for chak $k=2$ by using generalized normal sheaf:

For any quadric hypersurface $V$ of dimension $n$, there is an $r$-plane through any point of $V$ for $r \leq n / 2$.

Now we will consider the family of complete intersections containing $r$-planes through all the points of the complete intersections. Let $r, n$ and $N$ be integers with $1 \leq r<n<N$. Let $H, G$ and $X$ be as above. Now we will define $\tilde{H}, \tilde{G}$ and $\tilde{X}$ as $\tilde{H}=\left\{(z, V) \in \mathbf{P}_{k}^{N} \times H \mid z \in\right.$ $V\}, \tilde{G}=\left\{(z, L) \in \mathbf{P}_{k}^{N} \times G \mid z \in L\right\}$ and $\tilde{X}=\left\{(z, L, V) \in \mathbf{P}_{k}^{N} \times X \mid z \in L \subset V\right\}$. Then we have the following diagrams, where vertical maps $q: \tilde{G} \rightarrow G, \tilde{q}: \tilde{X} \rightarrow X$ and $p: \tilde{H} \rightarrow H$ are the second projections. Note that the maps $q$ and $\tilde{q}$ are $\mathbf{P}^{r}$-bundles.

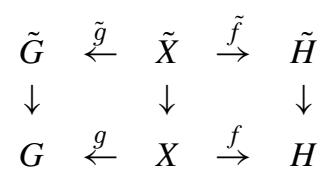

In this case we have

$$
\operatorname{dim} \tilde{X}-\operatorname{dim} \tilde{H}=(r+1)(N-r)-\sum_{j=1}^{N-n}\left(\begin{array}{c}
r+d_{j} \\
r
\end{array}\right)+r-n .
$$

Proposition 3.2. Under the above conditions, if $n=N-1$ and $d_{1}=2$, that is, a hyperquadric case, then $\tilde{f}$ is surjective.

PROOF. As in the proof of Theorem 3.1, we want to find a point $x \in \tilde{X}$ corresponding to $(z, L, V)$ such that $T_{\tilde{f}, x}: T_{x, \tilde{X}} \rightarrow T_{\tilde{f}(x), \tilde{H}}$ is surjective. From the infinitesimal theory of 
the previous section we obtain the following diagram of exact sequences:

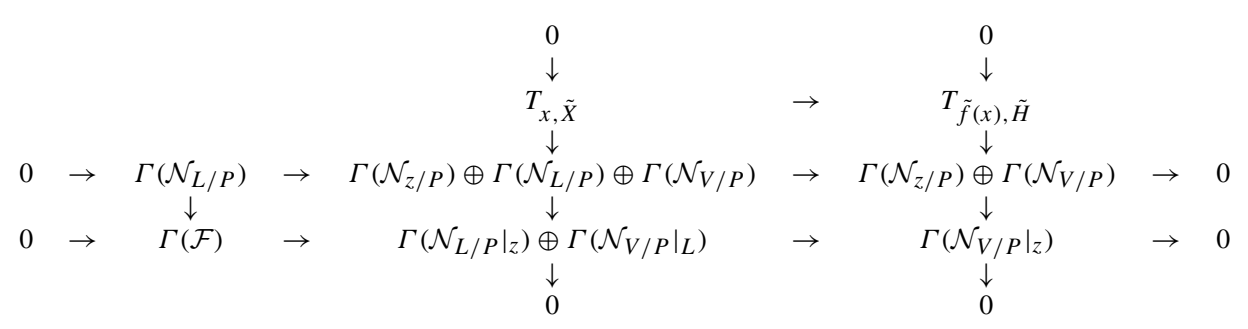

where $\mathcal{F}$ is the fibre product of $\left.\mathcal{N}_{L / P}\right|_{z}$ and $\left.\mathcal{N}_{V / P}\right|_{L}$ over $\left.\mathcal{N}_{V / P}\right|_{z}$. We have only to show that $\varphi: \Gamma\left(\mathcal{N}_{L / P}\right) \rightarrow \Gamma(\mathcal{F})$ is surjective. Now we take $x=(z, L, V) \in \tilde{X}$, where $L$ and $V$ are the same as in the proof of Theorem 3.1 and $z=(1: 0: \cdots: 0) \in P$. Note that $\Gamma\left(\mathcal{N}_{L / P}\right)=\Gamma\left(\mathcal{O}_{L}(1)\right)^{N-r}$ and $\Gamma(\mathcal{F}) \subset \Gamma\left(\left.\mathcal{N}_{L / P}\right|_{z}\right) \oplus \Gamma\left(\left.\mathcal{N}_{V / P}\right|_{L}\right)=k^{N-r} \oplus \Gamma\left(\mathcal{O}_{L}(2)\right)$. We will prove only for the $\mathrm{N}$ odd case since the even case is similar. Let us take bases as $\Gamma\left(\mathcal{N}_{L / P}\right) \cong \Gamma\left(\mathcal{O}_{L}(1)\right) \cdot \mathbf{e}_{1}^{\prime} \oplus \cdots \oplus \Gamma\left(\mathcal{O}_{L}(1)\right) \cdot \mathbf{e}_{N-r}^{\prime}$ and $\Gamma\left(\left.\mathcal{N}_{L / P}\right|_{z}\right) \oplus \Gamma\left(\left.\mathcal{N}_{V / P}\right|_{L}\right) \cong$ $k \cdot \mathbf{e}_{1} \oplus \cdots k \cdot \mathbf{e}_{N-r} \oplus \Sigma_{0 \leq i \leq j \leq r} k \cdot X_{i} X_{j}$. Let us express a basis of $\Gamma(\mathcal{F})$ in terms of a basis of $k \cdot \mathbf{e}_{1} \oplus \cdots k \cdot \mathbf{e}_{N-r} \oplus \Sigma_{0 \leq i \leq j \leq r} k \cdot X_{i} X_{j}$. In fact, a basis of $\Gamma(\mathcal{F})$ consists of the elements $\left\{\mathbf{e}_{1}, \ldots, \mathbf{e}_{N-r-1}, \mathbf{e}_{N-r}+X_{0}^{2}, X_{i} X_{j} \mid 0 \leq i \leq j \leq r, j \neq 0\right\}$. Under the notation we will describe the map $\varphi: \Gamma\left(\mathcal{N}_{L / P}\right) \rightarrow \Gamma(\mathcal{F}) \subset \Gamma\left(\left.\mathcal{N}_{L / P}\right|_{z}\right) \oplus \Gamma\left(\left.\mathcal{N}_{V / P}\right|_{L}\right)$. The composite map of $\varphi$ to the first direct summand $\Gamma\left(\mathcal{N}_{L / P}\right) \rightarrow \Gamma\left(\left.\mathcal{N}_{L / P}\right|_{z}\right)$ is written as $\Gamma\left(\mathcal{O}_{L}(1)\right)^{N-r} \rightarrow k^{N-r}$ by substituting a linear form by $X_{0}=1, X_{1}=\cdots=X_{r}=$ 0 , while the composite map of $\varphi$ to the second one $\Gamma\left(\mathcal{N}_{L / P}\right) \rightarrow \Gamma\left(\left.\mathcal{N}_{V / P}\right|_{L}\right)$ is written as $\left[0, \ldots, 0, X_{r}, \ldots, X_{0}\right]: \Gamma\left(\mathcal{O}_{L}(1)\right)^{r+1} \rightarrow \Gamma\left(\mathcal{O}_{L}(2)\right)$ by [2]. In this way the elements $X_{j} \mathbf{e}_{N-r-i+1}^{\prime}$ in $\Gamma\left(\mathcal{N}_{L / P}\right) \cong \Gamma\left(\mathcal{O}_{L}(1)\right) \cdot \mathbf{e}_{1}^{\prime} \oplus \cdots \oplus \Gamma\left(\mathcal{O}_{L}(1)\right) \cdot \mathbf{e}_{N-r}^{\prime}$ is mapped to the elements $X_{i} X_{j}$ in $\Gamma(\mathcal{F}) \subset k \cdot \mathbf{e}_{1} \oplus \cdots k \cdot \mathbf{e}_{N-r} \oplus \Sigma_{0 \leq i \leq j \leq r} k \cdot X_{i} X_{j}$ for $0 \leq i \leq j \leq r$, $j \neq 0, X_{0} \mathbf{e}_{N-r-i+1}^{\prime}-X_{i} \mathbf{e}_{N}^{\prime}$ to $\mathbf{e}_{i}$ for $1 \leq i \leq N-r-1$, and $X_{0} \mathbf{e}_{N}^{\prime}$ to $\mathbf{e}_{N-r}+X_{0}^{2}$. Hence the assertion is proved.

THEOREM 3.3. Under the above conditions, if $\operatorname{dim} \tilde{X} \geq \operatorname{dim} \tilde{H}$, then $\tilde{f}$ is surjective.

Proof. We may assume either $n \neq N-1$ or $d_{1} \neq 2$ by Proposition 3.2. Let us put $\varepsilon=$ $\operatorname{codim}(\tilde{f}(\tilde{X}), \tilde{H})$. We will show that $\varepsilon=0$. Let $g_{1}=q \circ \tilde{g}: \tilde{X} \rightarrow \tilde{G} \rightarrow G$. Fix an $r$-plane $L \in G$. Then $\tilde{H}^{\prime}=\tilde{f}\left(g_{1}^{-1}(L)\right)$ is a subvariety of $\tilde{H}$ with $\operatorname{dim} \tilde{H}^{\prime}=\operatorname{dim} \tilde{X}-\operatorname{dim} \tilde{G}+r(=$ $\operatorname{dim} X-\operatorname{dim} G+r)$. There is an irreducible component $\tilde{Y}$ of $\tilde{f}^{-1}\left(\tilde{H}^{\prime}\right)$ such that $\tilde{f}(\tilde{Y})=\tilde{H}^{\prime}$ and $\operatorname{dim} \tilde{Y}=\operatorname{dim} \tilde{f}^{-1}\left(\tilde{H}^{\prime}\right)$. Then we have $\operatorname{dim} \tilde{Y} \geq \operatorname{dim} \tilde{H}^{\prime}+\operatorname{dim} \tilde{X}-\operatorname{dim} \tilde{f}(\tilde{X})=2 \operatorname{dim} \tilde{X}-$ $\operatorname{dim} \tilde{G}-\operatorname{dim} \tilde{H}+r+\varepsilon$. Let $g_{1}^{\prime}: \tilde{Y} \rightarrow G^{\prime}=g_{1}(\tilde{Y})(\subseteq G)$ be a restriction of $g_{1}$. Note that $L \cap L^{\prime} \neq \phi$ for any $L^{\prime} \in G^{\prime}$. Let $G_{s}$ be a closed subscheme of $G$ which parametrizes $r$-planes $L^{\prime}$ such that $\operatorname{dim} L \cap L^{\prime} \geq s$. Let $s$ be the maximal integer such that $G^{\prime}$ is contained in $G_{s}$. Fix $L^{\prime} \in G^{\prime}$ such that $\operatorname{dim} L \cap L^{\prime}=s$. Let $S=\left\{V \in H \mid L \cup L^{\prime} \subset V\right\}$. Let $\tilde{S}=\{(z, V) \in$ $\left.\tilde{H} \mid z \in L \cap L^{\prime}, L \cup L^{\prime} \subset V\right\}$. Now we have $\operatorname{dim} g_{1}^{\prime-1}\left(L^{\prime}\right) \leq \operatorname{dim} \tilde{S}=\operatorname{dim} S+s$. From the 
proof of Theorem 3.1 we have $\operatorname{dim} S=2 \operatorname{dim} X-2 \operatorname{dim} G-\operatorname{dim} H+\sum_{j=1}^{N-n}\left(\begin{array}{c}s+d_{j} \\ s\end{array}\right)$. As in the proof of Theorem 3.1 we see

$$
\begin{aligned}
\operatorname{dim} \tilde{Y} & \leq \operatorname{dim} g_{1}^{\prime-1}\left(L^{\prime}\right)+\operatorname{dim} G^{\prime} \leq \operatorname{dim} S+s+\operatorname{dim} G_{s} \\
& =2 \operatorname{dim} X-\operatorname{dim} G-\operatorname{dim} H+s-P(s) \\
& =2 \operatorname{dim} \tilde{X}-\operatorname{dim} \tilde{G}-\operatorname{dim} \tilde{H}-r+n+s-P(s),
\end{aligned}
$$

where $P(s)=(s+1)(N-2 r+s)-\sum_{j=1}^{N-n}\left(\begin{array}{c}s+d_{j} \\ s\end{array}\right)$. Thus we see $\varepsilon \leq n-2 r+s-P(s)$. Let $Q(s)=P(s)-s-n+2 r$ for $s=0, \ldots, r$. Then we have $Q(0)=0, Q(r)=$ $\operatorname{dim} \tilde{X}-\operatorname{dim} \tilde{H} \geq 0$ and $\Delta^{2} Q(s)=\Delta^{2} P(s) \geq 0$ by the proof of Theorem 3.1 because $V$ is not a hyperquadric. Thus we have $\varepsilon \leq Q(s) \leq 0$, so $\varepsilon=0$. Hence the assertion is proved.

REMARK 3.4. If $n \geq 2 r$, there is a smooth complete intersection $V$ of $\mathbf{P}_{k}^{N}$ with $\operatorname{dim} V=n$ containing an $r$-plane $L$. On the other hand, a smooth complete intersection $V$ of $\mathbf{P}_{k}^{N}$ with $\operatorname{dim} V=n<2 r$ contains no $r$-planes.

Indeed, we firstly show a generic member $V=F_{1} \cap \cdots \cap F_{N-n}$ is smooth outside $L$, where $F_{j} \in\left|\mathcal{O}_{P}\left(d_{j}\right)-L\right|$ for $j=1, \ldots, N-n$, by the blowing up of $\mathbf{P}_{k}^{N}$ with centre $L$. In order to show a generic member $V$ is smooth along $L$, it is enough to take a smooth complete intersection $V$ defined by $\sum_{j=r+1}^{N} X_{j} f_{i j}=0$ for $i=1, \ldots, N-n$, where $f_{i j}=X_{j-i}^{d_{i}-1}$ if $0 \leq j-i \leq r, f_{i j}=0$ otherwise. Here $\mathbf{P}_{k}^{N}=\operatorname{Proj} k\left[X_{0}, \ldots, X_{N}\right]$ and $L: X_{r+1}=\cdots=$ $X_{N}=0$. Hence the first assertion is proved. The second assertion, in case char $k=0$, is an easy consequence of Lefschetz theorem. In case char $k>0$, assume that there exists a pair of a smooth complete intersection $V$ and an $r$-plane $L$. Let us consider the completion of the ring of Witt vectors $\widehat{W(k)}$. Here $\widehat{W(k)}$ is a complete local domain with residue field $\widehat{W(k)} / \widehat{\mathfrak{m}(k)} \cong$ $k$ and its quotient field has characteristic zero, see, e.g., [8]. Since $\mathrm{H}^{1}\left(\mathcal{N}_{L / V / \mathbf{P}_{k}^{N}}\right)=0$, the pair can be lifted a pair of liftings over $\widehat{W(k)}$ by Lemma 2.3 , which contradicts the assertion for char $k=0$.

Proof OF COROllary 1.1 AND COROllary 1.2. Corollary 1.1 follows from Proposition 3.2 and Theorem 3.3. For fixed integers $\left(d_{1}, \ldots, d_{N-n}\right)$, we write $X_{r}$ for an incidence correspondence $X \subset G \times H$ in case $G=G(r+1, N+1)$ under the notation before Theorem 3.1, and write $f_{r}: X_{r} \subset G \times H \rightarrow H$ for $f: X \subset G \times H \rightarrow H$. A scheme $H_{0}$ of the smooth complete intersections is an open set of a rational variety $H$. Note that $H_{r}=H_{0} \cap f_{r}\left(X_{r}\right)$. Then we have $H_{r}$ is not empty for $n \geq 2 r$ from Remark 3.4. Since $X_{r}$ is rational, $H_{r}$ is a unirational subvariety of the expected dimension by Theorem 3.1. Hence we have Corollary 1.2 .

ACKNOWLEDGEMENT. The starting draft of this paper was written up in 1982 as an enlarged version of my master thesis [7]. I have completely rewritten at present for a publication from encouragement by Takuzo Okada and Eiichi Sato. 


\section{References}

[1] M. ARTIN, Lectures on deformations of singularities, Tata Institute of Fundamental Research, Lectures on Mathematics 54, Bombay (1976).

[2] W. BARTh and A. VAN DE VEn, Fano-varieties of lines of hypersurfaces, Arch. Math. 31 (1978), 96-104.

[ 3 ] R. Beheshti, Linear subvarieties on hypersurfaces, Int. Math. Res. Not. 49 (2005), 3055-3063.

[ 4 ] C. BORCEA, Deforming varieties of $k$-planes of projective complete intersection, Pacific J. Math. 143 (1990), $25-36$.

[ 5 ] A. Collino, Lines on quartic threefolds, J. London Math. Soc. (2) 19 (1979), 257-267.

[6] A. Grothendieck, Fondements de la Géometrie Algébrique, Séminaire Bourbaki, Sécretariat Math. (1962).

[ 7 ] C. MiYAZAKI, Fano varieties of $r$-dimensional linear subspaces of hypersurfaces (in Japanese), Master Thesis (1982), Waseda University.

[ 8 ] D. MUMFORD, Lectures on curves on an algebraic surface, Annals of Math. Studies 59 (1966), Princeton UP.

[9] K. PARANJAPE and V. SRINIVAS, Unirationality of the general complete intersection of small multidegree, Flips and abundance for algebraic threefold by J. Kollár, Asterisque 211, 1992.

[10] A. PredonZan, Intorno agli $S_{k}$ giacenti sulla varietàinterszione completa di più forme, Atti Accad. Naz. Lincei. Rend. Cl. Sci. Fis. Mat. Nat. (8) 5 (1948), 238-242.

[11] B. R. Tennison, On the quartic threefold, Proc. London Math. Soc. 29 (1974), 714-734.

Present Address:

Department of Mathematics, Faculty of Education, KUMAMOTO UNIVERSITY, KUROKAMI 2-40-1, CHUO-KU, KUMAMOTO 860-8555, JAPAN.

e-mail: cmiyazak@educ.kumamoto-u.ac.jp 